\title{
Initial State Dependence in Multielectron Threshold Ionization of Atoms
}

\author{
Agapi Emmanouilidou, ${ }^{1}$ Peijie Wang, ${ }^{2, *}$ and Jan M. Rost ${ }^{2}$ \\ ${ }^{1}$ ITS, University of Oregon, Eugene, Oregon 97403-5203, USA \\ ${ }^{2}$ Max Planck Institute for the Physics of Complex Systems, Nöthnitzer Strasse 38, D-01187 Dresden, Germany
}

(Received 9 October 2007; published 13 February 2008)

It is shown that the geometry of multielectron threshold ionization in atoms depends on the initial configuration of bound electrons. The reason for this behavior is found in the stability properties of the classical fixed point of the equations of motion for multiple threshold fragmentation. Specifically for three-electron breakup, apart from the symmetric triangular configuration also a breakup of lower symmetry in the form of a $\mathrm{T}$ shape can occur, as we demonstrate by calculating triple photoionization for the lithium ground and first excited states. We predict the electron breakup geometry for threshold fragmentation experiments.

DOI: 10.1103/PhysRevLett.100.063002

Three-body Coulomb dynamics, in particular, twoelectron atoms, are very well studied in the energy regime of single as well as double ionization [1-4]. Much less is known about correlated dynamics in four-body Coulomb systems, more precisely on differential observables for fragmentation of a three-electron atom in its nucleus and all electrons [5-8]. A recent experiment provides for the first time detailed information in terms of differential cross sections on the angular and energetic breakup parameters of three electrons following impact double ionization of Helium [9]. For small excess energies $E$ (each continuum electron carries away about $9 \mathrm{eV}$ energy), it was found that the electrons form an equilateral triangle upon breaking away from the nucleus. This is expected in accordance with Wannier's theory [10], quantified for three electrons in [11]. There, it is shown that the fixed point of classical dynamics, through which full fragmentation near threshold $E=0$ should proceed, is given for a three-electron atom by an equilateral triangle with the nucleus in the center and the electrons at the corners.

In two-electron atoms the corresponding fixed point implies a collinear escape of the electrons in opposite directions $[10,12]$. The normal mode vibration about this collinear configuration is stable. This is in marked contrast to the three-electron case, where the triangular configuration is linked to two unstable, degenerate normal modes $[11,13]$.

We will show that the latter property has the consequence that the preferred final geometry of the three escaping electrons becomes initial state dependent and can change between an equilateral triangle and a less symmetric T-shaped escape. While the former is realized, e.g., in electron impact double ionization of helium, the latter should be seen in triple photoionization of lithium. These are only two prominent examples. The general pattern and the reason for it will be detailed below.

Because of the scaling of the Coulomb potential, states of finite total angular momentum $L$ will all behave like the $L=0$ state close to threshold which is therefore sufficient to consider [14]. In hyperspherical coordinates with the
PACS numbers: 32.80.Fb, 05.45.-a, 34.80.Dp

radial variable $w$ instead of the hyperradius $r=w^{2}$, the Hamiltonian for a three-electron atom with total angular momentum $L=0$ reads

$$
h=\frac{p_{w}^{2}}{8 w^{2}}+\frac{\Lambda^{2}}{2 w^{4}}+\frac{C(\boldsymbol{\Omega})}{w^{2}},
$$

where $\boldsymbol{\Omega}=\left(\alpha_{1}, \alpha_{2}, \theta_{1}, \theta_{2}\right)^{\dagger}$ contains all angular variables describing the positions of the electrons on the hypersphere of radius $r$ and $\Lambda$, the so-called grand angular momentum operator [15], is a function of $\boldsymbol{\Omega}$ and all conjugate momenta. The total Coulomb interaction $V=C / r$ acquires in this form simply an angular dependent charge $C(\boldsymbol{\Omega})$. In terms of the familiar vectors $\mathbf{r}_{1}, \mathbf{r}_{2}, \mathbf{r}_{3}$ pointing from the nucleus to each electron, the hyperspherical coordinates are given by

$$
\begin{aligned}
r & =\left(r_{1}^{2}+r_{2}^{2}+r_{3}^{2}\right)^{1 / 2}, \\
\alpha_{1} & =\arctan \left(r_{1} / r_{2}\right), \\
\alpha_{2} & =\arctan \left(r_{2 e} / r_{3}\right), \\
\theta_{1} & =\arctan \left[\mathbf{r}_{1} \cdot \mathbf{r}_{2} /\left(r_{1} r_{2}\right)\right], \\
\theta_{2} & =\arctan \left[\mathbf{r}_{1} \cdot \mathbf{r}_{3} /\left(r_{1} r_{3}\right)\right],
\end{aligned}
$$

where $r_{2 e}=\left(r_{1}^{2}+r_{2}^{2}\right)^{1 / 2}$.

Threshold dynamics is governed by motion along the normal modes about the fixed point of the Hamiltonian $H=(h-E) w^{2}$ [16]. The special form of $H$ ensures that the dynamics remains regular approaching the fixed point radially, i.e., $w \rightarrow w^{*}=0$, while $\boldsymbol{\Omega}^{*}$ at the fixed point is defined through $\left.\boldsymbol{\nabla}_{\Omega} C(\boldsymbol{\Omega})\right|_{\mathbf{\Omega}=\mathbf{\Omega}^{*}}=\mathbf{0}$. The equations of motion can be expressed as a system of first order differential equations (ODE) $\dot{\boldsymbol{\Gamma}}=G \nabla_{\Gamma} H$ for the phase space vector $\boldsymbol{\Gamma}=\left(p_{w}, \boldsymbol{P}_{\mathbf{\Omega}}, w, \mathbf{\Omega}\right)^{\dagger}$, with

$$
\boldsymbol{G}=\left(\begin{array}{cc}
0 & -\mathbf{1}_{f} \\
\mathbf{1}_{f} & 0
\end{array}\right)
$$

a block matrix composed from 0 and unity matrices of dimension $f \times f$ [17], where $f$ is the number of degrees of freedom, here $f=5$. Since the differential equations are still singular at the fixed point $\left(w^{*}, \Omega^{*}\right)$ a change of the 
momentum variables $\boldsymbol{P}_{\Omega}$ conjugate to $\Omega$ is needed

$$
p_{\omega_{j}}=P_{\omega_{j}} / w
$$

as well as a new time variable $\tau$ related to the original time $t$ conjugate to the Hamiltonian Eq. (1) through $d t=$ $w^{3} d \tau$. Finally, the normal modes can be obtained from the modified ODE, $d \boldsymbol{\gamma} / d \tau=G \nabla_{\gamma} \tilde{H}$ by diagonalizing the matrix $\partial^{2} G \tilde{H} /\left.(\partial \boldsymbol{\gamma} \partial \boldsymbol{\gamma})\right|_{\gamma=\gamma^{*}}$, where $\boldsymbol{\gamma}$ refers to the new phase space variables with the (noncanonical) momenta from Eq. (4). The eigenvalues are the Liapunov exponents $\lambda_{j}$ and in the normal mode basis $\left\{\hat{\boldsymbol{u}}_{j}\right\}$ threshold dynamics assumes an oscillatorlike form of $\delta \boldsymbol{u}_{j}(\tau)=$ $\exp \left(\lambda_{j} \tau\right) \delta \boldsymbol{u}_{j}(0)$ with the unit vectors $\hat{\boldsymbol{u}}_{j}$

$$
\hat{\boldsymbol{u}}_{j}=\delta \boldsymbol{u}_{j}(0) /\left|\delta \boldsymbol{u}_{j}(0)\right|
$$

defining the normal mode basis. The $\delta \boldsymbol{\gamma}(\tau)=\boldsymbol{\gamma}-\boldsymbol{\gamma}^{*}$ are excursions of $\boldsymbol{\gamma}$ from their fixed point values $\boldsymbol{\gamma}^{*}$ and are expressed as a linear combination of the $\delta \boldsymbol{u}_{j}(\tau)$.

We recall briefly the familiar three-body breakup in a two-electron atom with hyperradius $r_{2 e}$ and the angles $\alpha_{1}$, $\theta_{1}$ defined as in Eq. (2). The charge corresponding to $C(\Omega)$ in Eq. (2) is for the two-electron problem

$$
\begin{aligned}
C_{2 e}\left(\alpha_{1}, \theta_{1}\right)= & -\frac{Z}{\sin \alpha_{1}}-\frac{Z}{\cos \alpha_{1}} \\
& +\frac{1}{\left[1-\sin \left(2 \alpha_{1}\right) \cos \theta_{1}\right]^{1 / 2}} .
\end{aligned}
$$

The fixed point analysis reveals a pair of unstable $\lambda_{1 / 2}=$ $-\lambda_{0} \pm \lambda$ and stable $\lambda_{3 / 4}=-\omega_{0} \pm i \omega$ Liapunov exponents $\left(\lambda_{0}, \omega_{0}, \lambda, \omega>0\right)$ with a shift $\left(-\lambda_{0}\right.$ and $-\omega_{0}$, respectively), compared to standard symplectic dynamics. The shift formally arises through the noncanonical transformation of the momentum variables Eq. (4) necessary to obtain normal mode motion about the singular fixed point $w^{*}=0$.

The resulting eigenvectors $\hat{\boldsymbol{u}}_{i}$ reveal orthogonal motion along $\theta_{1}$ and $\alpha_{1}$, i.e., any phase space vector $\delta \gamma_{\alpha_{1}}(\tau)$ describing linearized motion in the subspace spanned by $\mathbf{p}_{\alpha_{1}}, \boldsymbol{\alpha}_{1}$, can be expressed as a linear combination of two eigenvectors $\delta \gamma_{\alpha_{1}}=a_{\gamma_{\alpha}} \exp \left(\lambda_{1} \tau\right) \hat{\boldsymbol{u}}_{1}+b_{\gamma_{\alpha}} \exp \left(\lambda_{2} \tau\right) \hat{\boldsymbol{u}}_{2}$. An analogous relation holds for linearized motion in the subspace $\mathbf{p}_{\theta_{1}}, \boldsymbol{\theta}_{1}$, realized through two different eigenvectors, $\delta \boldsymbol{\gamma}_{\theta_{1}}=a_{\gamma_{\theta}} \exp \left(\lambda_{3} \tau\right) \hat{\boldsymbol{u}}_{3}+b_{\gamma_{\theta}} \exp \left(\lambda_{4} \tau\right) \hat{\boldsymbol{u}}_{4}$. Hence, $\delta \gamma_{\theta_{1}}(\tau) \cdot \delta \gamma_{\alpha_{1}}(\tau)=0$ at all times $\tau$. The coincidence of the eigenspaces with the respective dynamics of $\theta_{1}$ and $\alpha_{1}$ has the important consequence that the fixed point value $\theta_{1}=\pi$ is preserved through its relation to the stable eigenmode while all energy sharings occur through their relation to the unstable eigenmode along $\alpha_{1}$.

This coincidence of normal modes with subspaces of observables is special to two-electron atoms and does not hold for more electrons. Moreover, for three-electron atoms, a new feature emerges in threshold dynamics, namely, the existence of two degenerate pairs of unstable normal modes with Liapunov exponents $\lambda_{1 / 2}=\lambda_{3 / 4}=$ $-\lambda_{0} \pm \lambda$. Note that the fragmentation dynamics close to the fixed point will take place in the phase space of the two unstable normal modes with an arbitrary linear combination of the two eigenvectors $\hat{\boldsymbol{u}}_{1}$ and $\hat{\boldsymbol{u}}_{3}$ belonging to the two (equal) positive Liapunov exponents $\lambda_{1}=\lambda_{3}=\lambda_{+}$,

$$
\delta \boldsymbol{\gamma}(\tau)=\exp \left(\lambda_{+} \tau\right)\left(c_{1} \hat{\boldsymbol{u}}_{1}+c_{3} \hat{\boldsymbol{u}}_{3}\right),
$$

which allows for flexibility in the four-body breakup, as we will see.

One sees directly from Table I that unlike the twoelectron case the normal mode dynamics about the fixed point for geometrical angles $\theta_{i}$ and hyperangles $\alpha_{i}$ is not separated, i.e., $\delta \gamma_{\theta_{i}}(\tau) \cdot \delta \gamma_{\alpha_{i}}(\tau) \neq 0$. Even more importantly, all phase space variables are linked to the unstable normal modes. Hence, the fixed point geometry does not provide necessarily a preference for the final angles of the electrons. On the other hand, this opens the way for the initial state to have an influence on the final observables, even close to threshold.

Relevant for threshold ionization is the spatial electron distribution at the time (we label it $\tau=0$ ) when all electrons to be ionized have received enough energy (through collisions) to leave the atom. We call this distribution the transient threshold configuration (TTC).

In a two-electron atom, the necessary energy transfer between the two electrons leading to three-body fragmentation happens through a single collision. At this time $\tau=$ 0 both electrons are naturally close together, so that $\delta \alpha_{1}(0) \approx 0$ holds and defines the TTC, independent of the initial bound electron configuration.

In a three-electron atom, the situation is more complicated since at least two collisions are necessary to distribute the energy among the electrons so that all of them can escape. For triple photoionization of lithium we know from classical calculations that the $1 s$ photoelectron (3), which has absorbed the photon initially, collides immediately with the other $1 s$ electron (2) and subsequently (about 60 attoseconds later) either electron 2 or the photoelectron itself collides with the $2 s$ electron (1). This can be expressed with the two collision sequences $s_{1}=(32,21)$ and $s_{2}=(32,31)[18]$. The time delay of the second collision,

TABLE I. The two eigenvectors $\hat{\boldsymbol{u}}_{i}$ belonging to the positive Liapunov exponent $\lambda>0$ in the basis of the phase space variables from Eq. (2).

\begin{tabular}{lcc}
\hline \hline Basis & $\hat{\boldsymbol{u}}_{1}$ & $\hat{\boldsymbol{u}}_{3}$ \\
\hline$\delta \alpha_{1}$ & $-8.22 \times 10^{-3}$ & $2.67 \times 10^{-1}$ \\
$\delta p_{\alpha_{1}}$ & $-2.97 \times 10^{-2}$ & $9.64 \times 10^{-1}$ \\
$\delta \alpha_{2}$ & $-1.81 \times 10^{-1}$ & 0 \\
$\delta p_{\alpha_{2}}$ & $-9.83 \times 10^{-1}$ & 0 \\
$\delta \theta_{1}$ & $7.12 \times 10^{-3}$ & 0 \\
$\delta p_{\theta_{1}}$ & $6.57 \times 10^{-3}$ & $-4.46 \times 10^{-3}$ \\
$\delta \theta_{2}$ & $3.33 \times 10^{-3}$ & $7.40 \times 10^{-3}$ \\
$\delta p_{\theta_{2}}$ & $-2.75 \times 10^{-4}$ & $8.92 \times 10^{-3}$ \\
\hline \hline
\end{tabular}



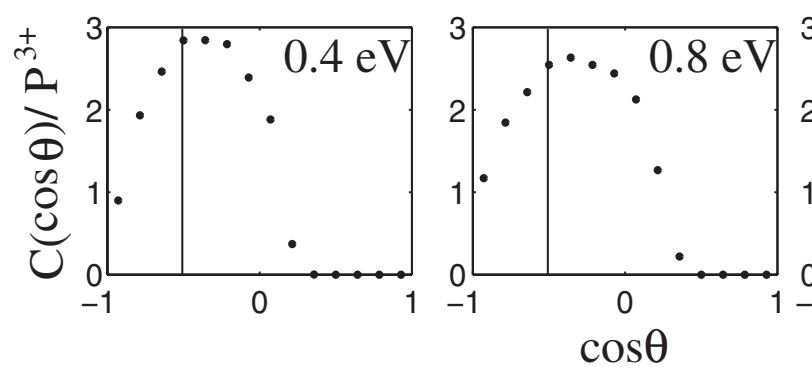

FIG. 1. Probability to find the angle $\theta$ between two electrons for triple ionization from the initial $\operatorname{Li}\left(1 s 2 s^{2}\right)$ state for excess energies above the triple ionization threshold as indicated. The preferred breakup geometry is the equilateral triangle. respectively, is due to the "distance" of the $2 s$ shell from the $1 s$ shell. It leaves an asymmetric situation after the second collision when the transient threshold configuration is reached at $\tau=0$. While the two electrons participating in the last collision are close to each other, the third one is further away. Concentrating on the collision sequence (32, 21 ), this implies in terms of distances to the nucleus

$$
r_{1} \approx r_{2} \neq r_{3}
$$

Hence, $\delta \alpha_{1}(0) \approx 0$ while $\delta \alpha_{2}(0) \neq 0$. This can be easily accommodated with a suitable linear combination choosing, e.g., $c_{1}=1 / a$ and $c_{2}=-1 / b$ in Eq. (7), where $a$ and $b$ are the coefficients of $\delta \alpha_{1}$ with respect to $\hat{\boldsymbol{u}}_{1}$ and $\hat{\boldsymbol{u}}_{3}$ (see first row of Table I).

The scenario described is still within the overall picture of threshold breakup in the spirit of Wannier and therefore in accordance with the power law $\sigma \propto\left(E / E_{0}\right)^{\alpha}$ for the dependence of the total breakup cross section on the excess energy $E$ [20-22]. The exponent $\alpha=\left(\lambda_{1}+\lambda_{3}\right) /\left(2 \lambda_{r}\right)=$ 2.162, where $\lambda_{r}=2.5088$ is the radial Liapunov exponent and $\lambda_{1}=\lambda_{3}=5.4240$. Yet, the TTC with unequal distances of the electrons to the nucleus breaks the complete symmetry among the electrons. This, in turn, leads to a preferred final geometry of the three electrons since essentially with the third electron being far away, the geometry determination reduces to that of two electrons escaping in an equivalent way as in the two-electron breakup, i.e., back-to-back with an angle of $\theta_{1}=\pi$. The question remains if there is a preferred angle between this electron pair escaping along a line and the third electron. Assuming for simplicity that $r_{3} \gg r_{1} \approx r_{2}$ and therefore $\sin \alpha_{2} \approx 0$ holds (the opposite case would lead to the same result), we can expand $C(\Omega)$ in Eq. (1) in powers of $\eta \equiv \alpha_{2}$

$$
C(\Omega) \approx \eta^{-1} \sum_{n=0}^{3} c_{n} \eta^{n} .
$$

One can show that to lowest order in $\eta$, the problem to find a stable configuration is that of the two-electron system (here with nuclear charge $Z=3$ ) with the well-known solution $\theta_{1}^{* *}=\pi$ and $\alpha_{1}^{* *}=\pi / 4$ [10]. These values minimize $c_{2}$ for any value $\theta_{2}$. Its value $\theta_{2}^{* *}=\pi / 2$ is determined from $\partial c_{3} / \partial \theta_{2}=0$ which is a stable solution. [The $\Omega^{* *}$ is used for the fixed point solution of $C(\Omega)$ when $r_{3} \gg$ $r_{1} \approx r_{2}$ to distinguish it from the $\Omega^{*}$ global fixed point solution of $C(\Omega)$ mentioned previously in the Letter.] This constitutes the $\mathrm{T}$ shape of the three electrons as a preferred asymptotically $(\eta \rightarrow 0)$ stable geometrical configuration within the globally unstable two-dimensional subspace spanned by $\hat{\boldsymbol{u}}_{1}, \hat{\boldsymbol{u}}_{3}$. The fragmentation dynamics in the subspace is completely degenerate-hence, we have looked on a "higher order" correction, which would give a preference within the degenerate subspace: that is the asymptotically stable $\mathrm{T}$ shape. Indeed, this configuration was found numerically close to threshold in triple photoionization [19].

One can double check this new insight into the role of the TTC of the electrons in the presence of degenerate Liapunov exponents by considering initial configurations which lead to different transient configurations.

For an initial excited state $\mathrm{Li}\left(1 s 2 s^{2}\right)$ the two collisions in which energy is transferred from the $(1 s)$ photoelectron to the two $2 s$ electrons happen close in time (and therefore in space). Hence, the transient threshold configuration after the second collision at $\tau=0$ is $r_{1} \approx r_{2} \approx r_{3}$ close to the fixed point and therefore giving preference to the symmetric breakup with a dominant angle $120^{\circ}$ between the electrons, similar to electron impact double ionization of helium.

Numerical results confirm this prediction as seen from Fig. 1 where the probability to find an angle $\theta$ between two electrons in triple photoionization of the initial $\operatorname{Li}\left(1 s 2 s^{2}\right)$ is shown for different excess energies. Clearly, the most likely angle is $\cos \theta=-1 / 2$ corresponding to $120^{\circ}$, in-
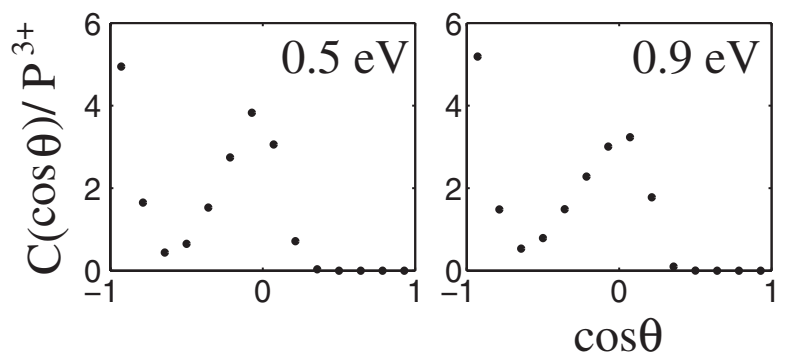

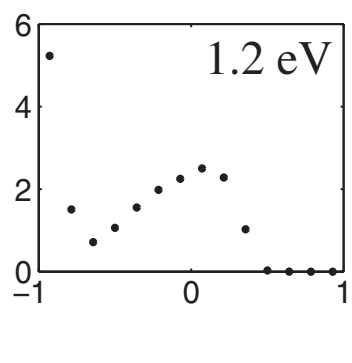

FIG. 2. Same as Fig. 1, but for triple ionization from the initial $\mathrm{Li}\left(1 s^{2} 2 s\right)$ ground state for excess energies above the triple ionization threshold as indicated. The preferred breakup geometry is the $T$ shape. 


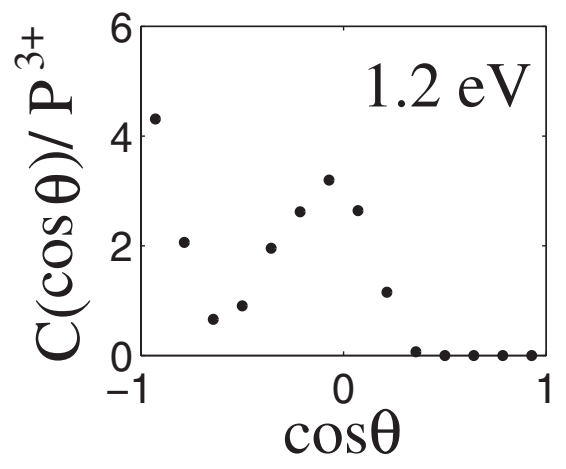

FIG. 3. Same as Fig. 1, but with a $2 s$ electron as photoelectron.

dicated by the thin vertical line. The method used to obtain the results in Figs. 1-3 is the classical trajectory Monte Carlo method detailed in [19].

On the other hand, for the ground state as initial state the TTC is asymmetric as previously described and we expect a final T shape geometry with peaks at $90^{\circ}$ and $180^{\circ}$. This is indeed the case, as can be seen in Fig. 2. As a final test we may use again the excited initial state $\operatorname{Li}\left(1 s 2 s^{2}\right)$ but take the $2 s$ electron as the photoelectron. This process is due to the smaller dipole coupling by more than an order of magnitude suppressed compared to the ionization with the $1 s$ electron absorbing the photon. However, here we use this only as an illustration for the initial state dependence of threshold ionization. According to our reasoning we have in this case (although it is the same initial state as before) a different TTC since the first collision of the photoelectron 1 happens with the $1 s$ electron 2 while later on the collision of the $1 s$ electron 2 with the $2 s$ electron 3 will take place. Consequently, for the TTC $r_{3} \approx r_{2} \neq r_{1}$ holds which is structurally identical to Eq. (8), the situation when a final T shape geometry appears. As can be seen in Fig. 3 the T shape indeed also emerges if the photoelectron comes from the $2 s$ shell.

To summarize, we predict that for three-electron breakup near threshold two preferred geometrical patterns for the electrons exist, namely, an equilateral triangle and a T shape. Which of them is realized depends on the transient threshold configuration, that is the spatial distribution of the electrons at the time when the energy among them is distributed such that all of them can escape. The transient

TABLE II. The relation of excitation process, initial configuration, and geometry of the electrons for various three-electron breakup processes close to threshold.

\begin{tabular}{lcc}
\hline \hline Impact by & Initial configuration & Breakup geometry \\
\hline Electron & $1 s^{2}(\mathrm{He})$ & $\Delta$ \\
Electron & $1 s 2 s(\mathrm{He})$ & $\perp$ \\
Photon & $1 s^{2} 2 s(\mathrm{Li})$ & $\perp$ \\
Photon & $1 s 2 s^{2}(\mathrm{Li})$ & $\Delta$ \\
Photon & $1 s 2 s 3 s(\mathrm{Li})$ & $\perp$ \\
\hline \hline
\end{tabular}

threshold configuration is strongly influenced by the initial state of the electrons, as has been discussed in detail for the case of lithium. A complete overview of the three-electron breakup pattern for three-electron systems is provided in Table II. Whenever the two electrons to which energy is transferred in the course of the fragmentation of the atom are in the same shell, a symmetric triangular geometry is expected. If the two electrons are from different shells, we expect a $T$ shape. Recent experimental results on electron impact double ionization of helium [9] are consistent with our predictions but do not provide sufficient information to consider our prediction as experimentally already confirmed. Based on the present consideration it is also possible to predict the breakup geometries and their realization dependent on the initial state for more than three electrons.

*Present address: The Beijing Key Laboratory for NanoPhotonics and Nano-Structure, Capital Normal University, Beijing 100037 People's Republic of China.

[1] G. Tanner, K. Richter, and J. M. Rost, Rev. Mod. Phys. 72, 497 (2000).

[2] J. S. Briggs and V. Schmidt, J. Phys. B 33, R1 (2000).

[3] T. N. Rescigno, M. Baertschy, W. A. Isaacs, and C.W. McCurdy, Science 286, 2474 (1999).

[4] C. W. Byun, N. N. Choi, M.-H. Lee, and G. Tanner, Phys. Rev. Lett. 98, 113001 (2007).

[5] J. Colgan and M. Pindzola, J. Phys. B 39, 1879 (2006).

[6] J. R. Götz, M. Walter, and J. S. Briggs, J. Phys. B 39, 4365 (2006).

[7] A. Emmanouilidou, Phys. Rev. A 75, 042702 (2007).

[8] A. W. Malcherek and J.S. Briggs, J. Phys. B 30, 4419 (1997).

[9] M. Dürr, A. Dorn, J. Ullrich, S. P. Cao, A. Czasch, A. S. Kheifets, J. R. Götz, and J. S. Briggs, Phys. Rev. Lett. 98, 193201 (2007).

[10] G. H. Wannier, Phys. Rev. 90, 817 (1953).

[11] H. Klar and W. Schlecht, J. Phys. B 9, 1699 (1976).

[12] J. M. Rost, Phys. Rev. Lett. 72, 1998 (1994).

[13] M. Y. Kuchiev and V. N. Ostrovsky, Phys. Rev. A 58, 321 (1998).

[14] J. M. Rost, Phys. Rep. 297, 271 (1998).

[15] F. T. Smith, Phys. Rev. 120, 1058 (1960).

[16] see Sec. 3.4 in [14].

[17] A. M. Ozorio de Almeida, Hamiltonian Systems: Chaos and Quantization (Cambridge University Press, Cambridge, U.K., 1988), p. 11.

[18] Note that for the present purpose we have relabeled the electrons compared to [19].

[19] A. Emmanouilidou and J. M. Rost, J. Phys. B 39, 4037 (2006).

[20] J. A. R. Samson and G. C. Angel, Phys. Rev. Lett. 61, 1584 (1988).

[21] R. Wehlitz, T. Pattard, M.-T. Huang, I. A. Sellin, J. Burgdörfer, and Y. Azuma, Phys. Rev. A 61, 030704(R) (2000).

[22] J. Bluett, D. Lukic, and R. Wehlitz, Phys. Rev. A 69, 042717 (2004). 